\title{
Acta Physiologiae Plantarum introduces Continuous Article Publishing
}

(C) Franciszek Górski Institute of Plant Physiology, Polish Academy of Sciences, Kraków 2014

Beginning with Volume 37 (2015) the journal Acta Physiologiae Plantarum will start using a new publication model called "Continuous Article Publishing."

All accepted articles will immediately be published in an issue. Springer's "Online First" mode, which uses the online publication of unpaginated articles, will become obsolete.

Instead of consecutive pagination of articles throughout a volume, each article will have an internal pagination beginning with page 1 . In addition, every article will have an Article Citation ID number. This unique article identifier will be visible on every page of an article and will replace the page numbers in the citation line.
Example

Old: Acta Physiol Plant (2014) 36:951-958

New: Acta Physiol Plant (2015) 37:1234

As a result, all articles will have their full and final citation information available as soon as the production process is complete. This change will not affect the production time from acceptance to online publication of an article, but since the articles will be published online immediately in their final form, the overall publication time for articles will become shorter.

We strongly believe that authors of Acta Physiologiae Plantarum will benefit from this change and that the new system will also simplify referencing of articles. 